\title{
Nuestra experiencia con el uso de cierre asistido al vacío.
}

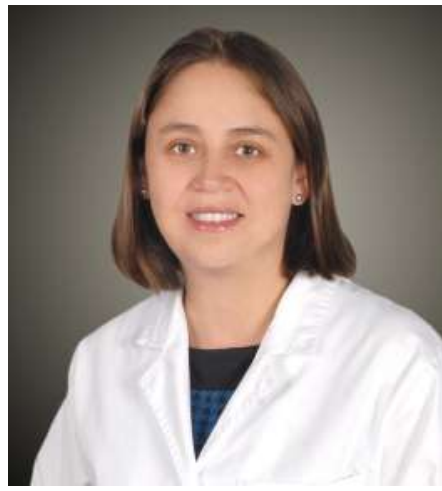

\author{
Dra. Claudia Alejandra Peláez Flores \\ Cirujana plástica, Miembro SBCPER, FICS \\ Centro médico de especialidades y rehabilitación CER \\ ale_pelaezf@hotmail.com.+59160163229 \\ Dr. Marco Antonio Paredes Nuñez. \\ Traumatólogo, Ortopedista, Miembro SBOLOT FICS \\ Centro médico de especialidades y rehabilitación CER
}

\section{"Our experience with the use of vacuum-assisted closure."}

\section{RESUMEN}

La aplicación de niveles controlados de presión subatmosférica han demostrado acelerar el proceso de curación de las heridas, aplicando este concepto, mostramos nuestra experiencia de 10 años de uso de esta terapia (VAC. Vaccumm-assited closure system) (Kinetic Concepts Inc., San Antonio TX).para tratamiento de diferentes tipos de heridas, a través de una revisión de casos interesantes.

Se estudiaron 162 pacientes con heridas complejas agudas y/ o crónicas, que comprometían diferentes partes del cuerpo, en todos los pacientes se realizó desbridamiento radical antes de aplicar la terapia de cierre asistido al vacío, todos recibieron presión negativa de forma intermitente y controlada y los cambios de la curación se realizaron cada 4 a 5 días.

El tratamiento duro como promedio 20 días, todos los pacientes que iniciaron la terapia con infección fueron curados al cabo de ella y no tuvimos complicaciones serias.

En nuestra experiencia de 10 años en el país, (BOLIVIA) creemos que la terapia cierre asistido al vacío, es el tratamiento de elección para todas aquellas heridas complejas, infectadas y que requieran cobertura, ya que pudimos comprobar la efectividad del sistema en todos los pacientes tratados, pues es de conocimiento general y demostrado ampliamente en la literatura, que el sistema Cierre Asistido al Vacío (CAV), incrementa el flujo capilar, estimula la granulación disminuyendo el tiempo requerido para la curación.

\section{ABSTRACT}

The application of controlled levels of subatmospheric pressure has been shown to accelerate the healing process of wounds, applying this concept, our experience of 10 years of use of this therapy (Vaccum-assited closure system) (Kinetic Concepts Inc., San Antonio TX). as treatment of different types of wounds, through a review of interesting cases.

We studied 162 patients with complex acute and / or chronic injuries that affected different parts of the body were studied, all patients underwent radical debridement before applying vacuum-assisted closure therapy, all received negative pressure intermittently and controlled and dressing changes were performed every 4 to 5 days.

The treatment lasted an average of 20 days, all the patients who started the therapy with infection were cured after it and we had no serious complications.

We present a review of some cases. In our experience of 10 years in the country, (BOLIVIA) we believe that VAC therapy is the treatment of choice for all those complex wounds, infected and requiring coverage, since we could verify the effectiveness of the system in all patients treated. It is of general knowledge and widely demonstrated in the literature, that the vacuum assisted closure system, increases capillary flow, stimulates granulation by decreasing the time required for healing.

\section{KEY WORDS}

Vacuum assisted closure, cierre asistido al vacío, heridas complejas, complex wounds 


\section{INTRODUCCIÓN}

La práctica de exponer una herida a los efectos de la presión atmosférica durante un período prolongado, para promover el desbridamiento y el tratamiento de las fracturas expuestas, fue descrita por primera vez, con éxito, por Fleischmann et al en 19931.

Posteriormente éste autor en dos documentos 2, 3 describe su experiencia en síndrome compartimental e infecciones agudas y crónicas en extremidad pélvica, con promedios variados en días de uso del sistema, así como de la conclusión del tratamiento reconstructivo. Muller 4 y Kovacs 5 describen su experiencia en heridas infectadas y en úlceras crónicas por radiación, respectivamente.

El uso de la presión negativa fue descrito por Barker 6 y otros investigadores en 1994, quienes hacen referencia a una "técnica de empaquetamiento al vacío", en pacientes con trauma abdominal en este tipo de tratamiento, los autores utilizaron compresas intraabdominales y un sistema de succión cerrada convencional.7,8

En 1995, Food and Drug Administration (FDA) de Estados Unidos aprobó el sistema V.A.C. (Vacuum Assisted Closure) para su utilización en pacientes diabéticos que presentaban lesiones en los pies, úlceras por presión, heridas quirúrgicas infectadas, colgajos e injertos, heridas traumáticas y otras lesiones de difícil cicatrización.

En el año 1997 Argenta y Morykwas (cirujanos plásticos) publicaron su experiencia clínica con el sistema de cierre asistido al vacío en pacientes con úlceras por presión y pie diabético, logrando demostrar su efectividad con resultados favorables.9

El sistema Cierre Asistido al Vacío (CAV), consta de una esponja porosa de poliuretanos, fijada a la herida por un adhesivo y conectada a la máquina de aspiración negativa a través de unos tubos de plástico.

La máquina permite controlar la presión negativa que se aplicará a la herida, que generalmente fluctúa entre - 75 a -175 mmHg. y también permite controlar si la presión aplicada será de forma intermitente o continua.

El objetivo del este trabajo es mostrar nuestra experiencia en la utilización de la terapia de cierre al vacío en Bolivia. A través de algunos casos interesantes.

\section{MATERIAL Y MÉTODOS}

Realizamos un estudio retrospectivo, de enero de 2008 a noviembre 2017 , donde tratamos a 162 pacientes con la terapia de cierre al vacío. Todos ellos con heridas complejas de diferente etiología.
De total de pacientes manejados, 92 pacientes presentaron fracturas expuestas Gustilo II - III, 12 pacientes con ulceras por presión, 4 infecciones post quirúrgicas de cadera, 2 infecciones post quirúrgicas en instrumentación de columna vertebral, 17 amputaciones de extremidades, 5 lesiones complejas de talón con pérdida de sustancia y cobertura a nivel de tendón de Aquiles, 12 fasciotomías, 2 complicaciones de abdominoplastia y 16 lesiones diversas ( 3 fascitis necrotizante, 2 quemaduras, 11 heridas infectadas).

No se tomó en cuenta como variables el sexo, edad del paciente, ni en caso de las infecciones el germen aislado.Por tanto todos pacientes, con las patologías antes descritas fueron incluidos en la muestra.

Antes de aplicar la bomba de presión negativa, se realizó en todos los pacientes, desbridamiento radical, todos recibieron presión negativa (oscila entre -90 y $-125 \mathrm{mmHg}$ ) continuo/intermitente y controlada.

Los pacientes con diagnóstico de fractura expuesta recibieron hasta 5 ciclos de terapia CAV, que fue el periodo más prolongado de tratamiento y los pacientes con complicaciones de abdominoplastia (seroma y dehiscencia de la herida) recibieron 1 ciclo de CAV, que es el periodo mínimo de utilización del sistema. Cada ciclo tiene 5 días de terapia, el resto de los pacientes (63) uso el sistema 304 ciclos, como lo muestra la Tabla 1 
TABLA N.1

\begin{tabular}{|c|c|c|c|c|c|c|c|}
\hline Diagnóstico & $\begin{array}{l}\mathrm{N}^{\circ} \text { de } \\
\text { casos }\end{array}$ & Varones & Mujeres & $\begin{array}{l}\mathrm{N}^{\circ} \text { de } \\
\text { ciclos }\end{array}$ & Terapia & Cobertura final & Complicaciones \\
\hline $\begin{array}{l}\text { Fractura expuesta } \\
\text { Gustilo II }\end{array}$ & 48 & 28 & 20 & 3 & Continua & $\begin{array}{l}\text { (32) Cierre primario } \\
\text { (16) Injerto de piel }\end{array}$ & No \\
\hline $\begin{array}{l}\text { Fractura expuesta } \\
\text { Gustilo III a }\end{array}$ & 36 & 31 & 5 & $4-5$ & Intermitente & $\begin{array}{l}\text { (29) injerto de piel } \\
\text { (7) Colgajo regional }\end{array}$ & No \\
\hline $\begin{array}{c}\text { Fractura expuesta } \\
\text { Gustilo III b }\end{array}$ & 8 & 5 & 3 & 5 & Intermitente & Colgajo regional & No \\
\hline Ulceras por Presión & 12 & 8 & 4 & $2-3$ & Intermitente & Colgajo regional & No \\
\hline $\begin{array}{c}\text { Infección post } \\
\text { quirúrgica de } \\
\text { cirugía de cadera }\end{array}$ & 4 & 2 & 2 & 3 & Continua & Cierre primario & No \\
\hline $\begin{array}{c}\text { Infección post } \\
\text { quirúrgica de } \\
\text { instrumentación } \\
\text { vertebral posterior }\end{array}$ & 2 & 2 & 0 & $3-4$ & Continua & Cierre primario & No \\
\hline $\begin{array}{l}\text { Amputación de } \\
\text { extremidades }\end{array}$ & 17 & 15 & 2 & 3 & Continua & Cierre primario & No \\
\hline $\begin{array}{l}\text { Lesiones complejas } \\
\text { de talón y tendón } \\
\text { de Aquiles }\end{array}$ & 5 & 5 & 0 & $3-4$ & Continua & Colgajo Sural & No \\
\hline Fasciotomías & 12 & 8 & 4 & 3 & Continua & $\begin{array}{l}\text { Cierre por tracción } \\
\text { cutánea + injerto de } \\
\text { piel }\end{array}$ & No \\
\hline $\begin{array}{c}\text { Complicación de } \\
\text { abdominoplastia } \\
\text { (seroma, } \\
\text { dehiscencia de } \\
\text { herida) }\end{array}$ & 2 & 0 & 2 & 1 & Continua & Cierre primario & No \\
\hline $\begin{array}{c}\text { Fascitis } \\
\text { necrotizante }\end{array}$ & 3 & 2 & 1 & $3-4$ & Continua & Injerto de piel & No \\
\hline Quemaduras & 2 & 1 & 1 & $1-2$ & Intermitente & Injerto de piel & No \\
\hline $\begin{array}{l}\text { Heridas diversas } \\
\text { infectadas }\end{array}$ & 11 & 6 & 5 & $2-3$ & Continua & Cierre primario & No \\
\hline
\end{tabular}




\section{CASO 1}

Paciente de 22 años de edad ingresa con cuadro de 18 horas de evolución posterior a sufrir accidente de tránsito (atropellado por un camión). Al momento de ingreso se evaluó la lesión (fractura expuesta de tibia izquierda Gustilo IIIB).

Se realizar la limpieza quirúrgica correspondiente y estabilización con fijadores externos; a las 24 horas se evalúan las partes blandas evidenciándose necrosis cutánea, realizamos desbridamiento y sistema de cierre asistido al vació (2 ciclos consecutivos).

Le evolución fue favorable y se realizó la cobertura con injerto de piel. La fractura consolido de manera satisfactoria con los fijadores externos.

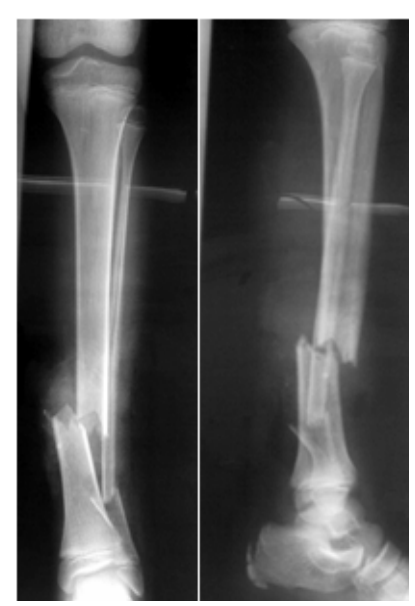

Foto 1

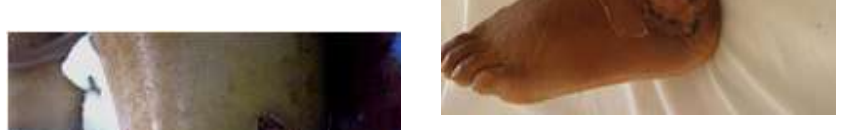

Foto 5

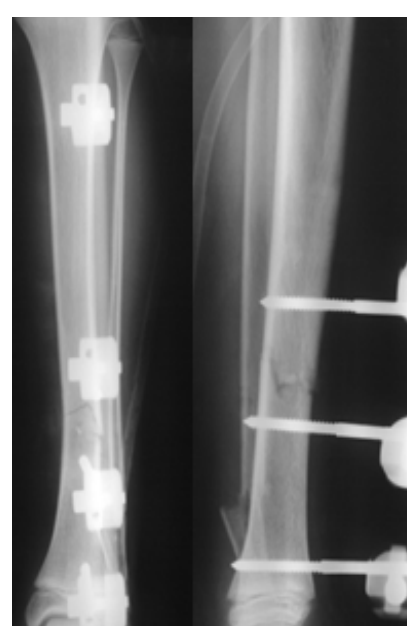

Foto 6
Foto 5 y 6: Cobertura cutánea con injerto de piel y radiografía de control

\section{CASO 2}

Paciente de 46 años de edad con instrumentación vertebral por vía posterior. A los 7 días de ser externado acude a la consulta por dolor en región operatoria, presencia de eritema y débito seroso.

Se realiza limpieza quirúrgica y toma de cultivo (reporte: S. Aureus sensible a Ciprofloxacina y Vancomicina) el paciente a las $24 \mathrm{Hrs}$. de tratamiento presenta reacción alérgica generalizada (se suspende el antibiótico).

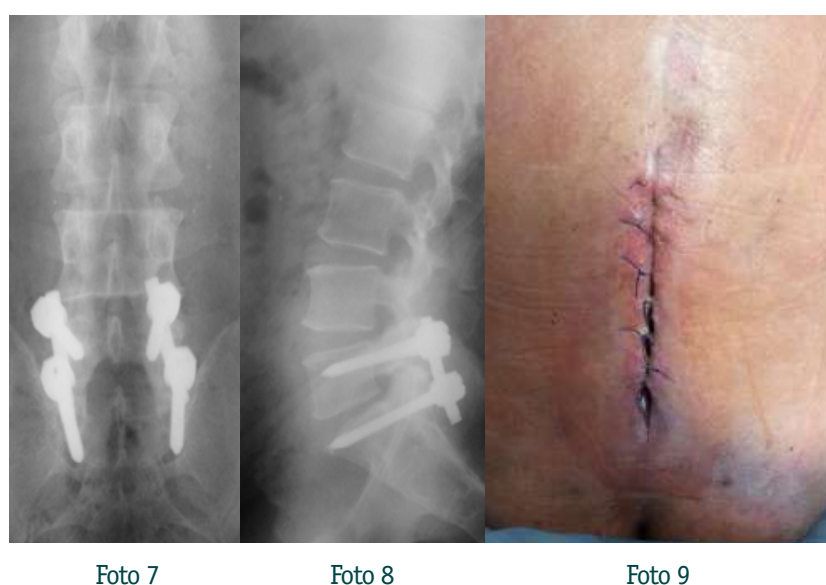

Foto 7,8,9 : Imágenes radiográficas de la instrumentación vertebral por vía posterior, a la derecha se observa clínicamente la herida quirúrgica con proceso infeccioso, dehiscencia y eritema. 


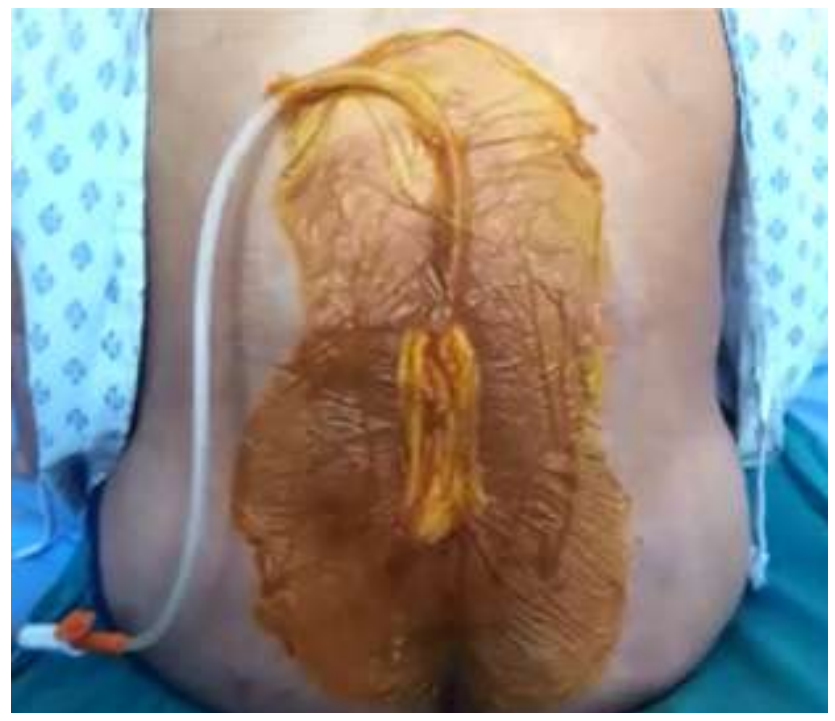

Foto 10

Foto 10 : Sistema de cierre asistido al vació en cirugía de columna vertebral

Se aplica sistema de cierre asistido al vació (3 ciclos de 5 días cada uno), la evolución fue favorable con buen tejido de granulación y cierre de la herida por segunda.

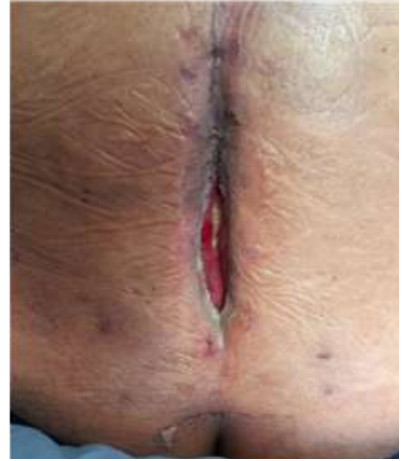

Foto 11

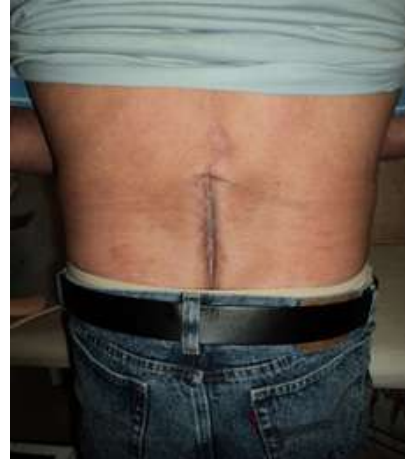

Foto 12
Foto 11 y 12: Herida después de 15 días con sistema de cierre asistido al vacío; buen tejido de granulación y cierre del espacio. A la derecha la herida completamente cicatrizada después de 4 meses.

\section{CASO 3}

Paciente de 18 años de edad que sufre traumatismos directo sobre brazo en extensión (le cae una biga de cemento desde una altura de aproximadamente 12 metros).

Ingresa al centro hospitalario con cuadro de 11 horas de evolución. Diagnóstico: amputación traumática de extremidad torácica izquierda y luxación de articulación glenohumeral izquierda.
El paciente es intervenido inmediatamente realizándose reducción de la luxación gleno-humeral, limpieza quirúrgica y aplicación de sistema de cierre asistido al vacío (se aplican 3 ciclos de 5 días cada uno).

La evolución fue satisfactoria con buen tejido de granulación; se realiza la cobertura definitiva con colgajo de pectoral mayor.

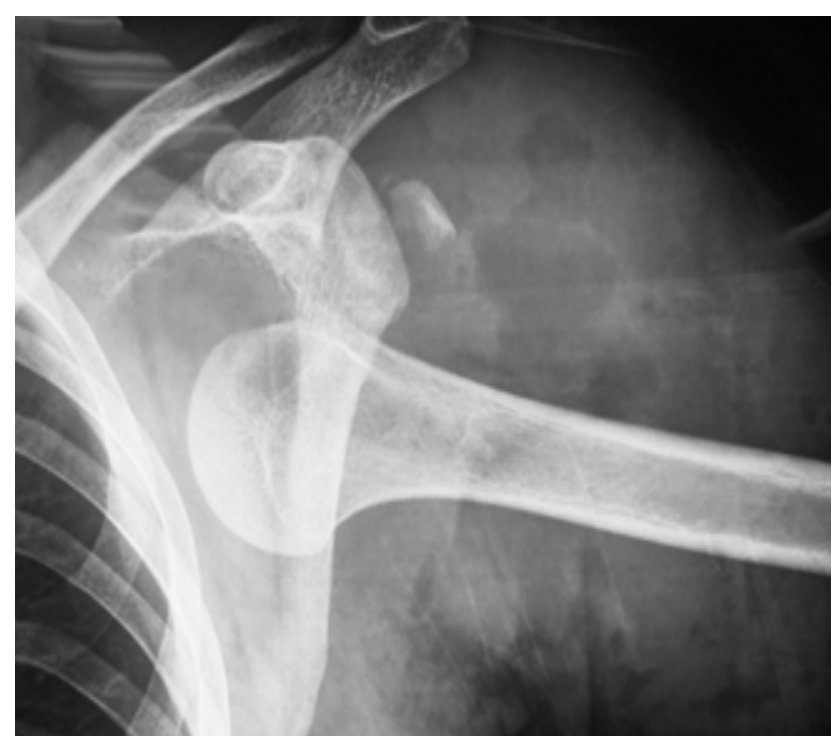

Foto 13

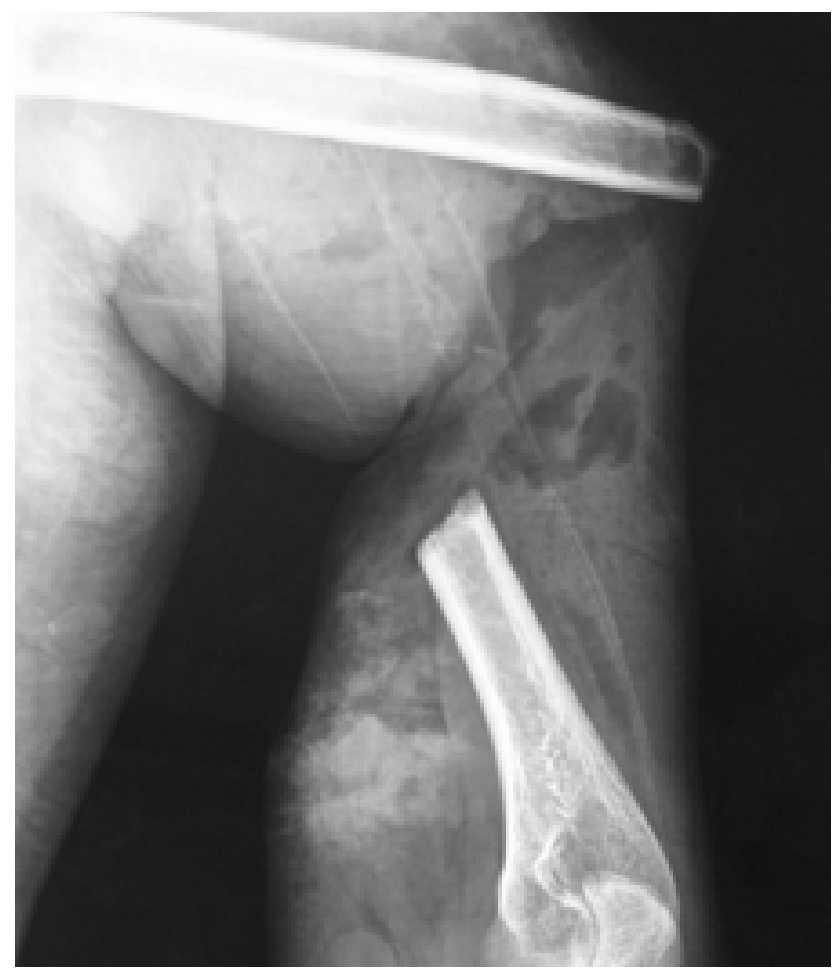

Foto 14

Foto 13 y 14: Imágenes radiográficas que corresponden al ingreso del paciente; se puede observar la luxación glenohumeral y la fractura medio diafisiaria en humero izquierdo 


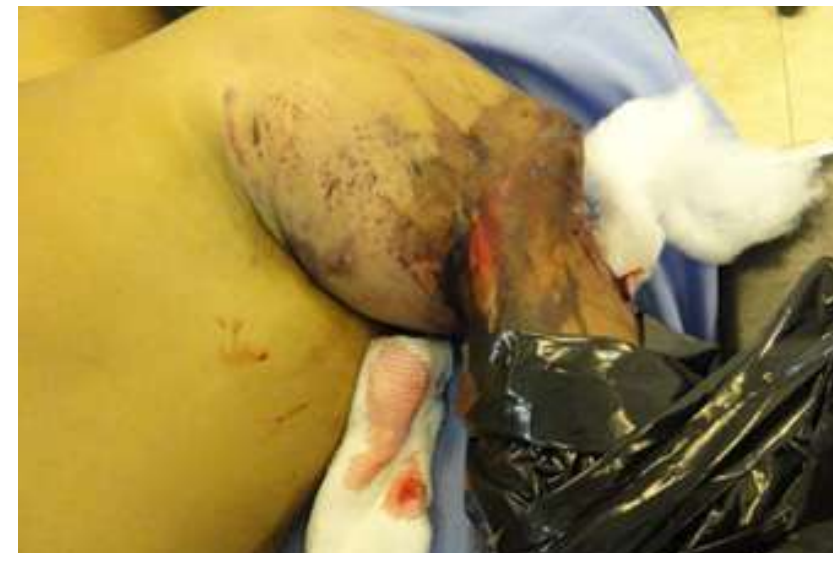

Foto 15

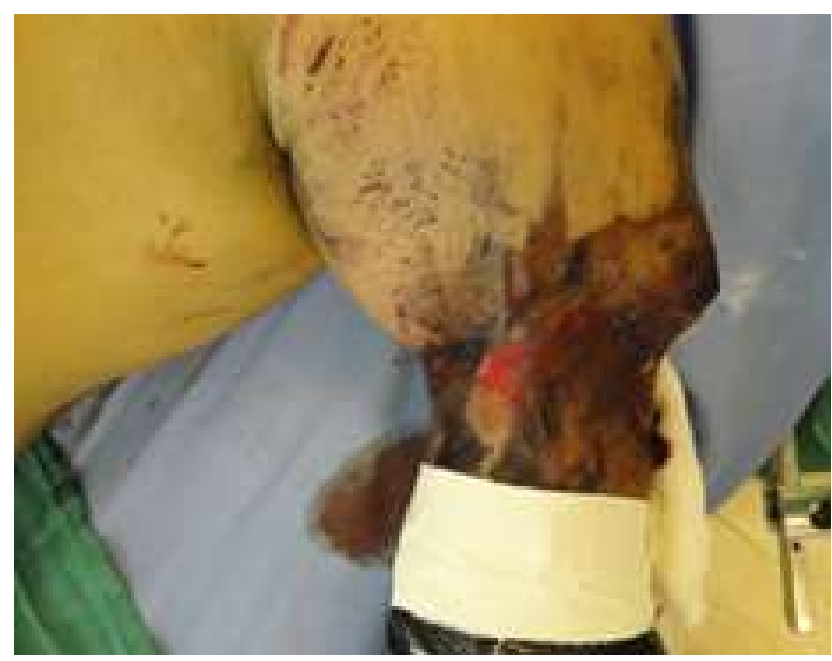

Foto 16

Foto 15 y 16 : Imágenes clínicas del ingreso del paciente; obsérvese el área de necrosis cutánea y que delimita el punto de la amputación traumática. El resto de la extremidad completamente necrótico se encuentra cubierto por una bolsa negra.

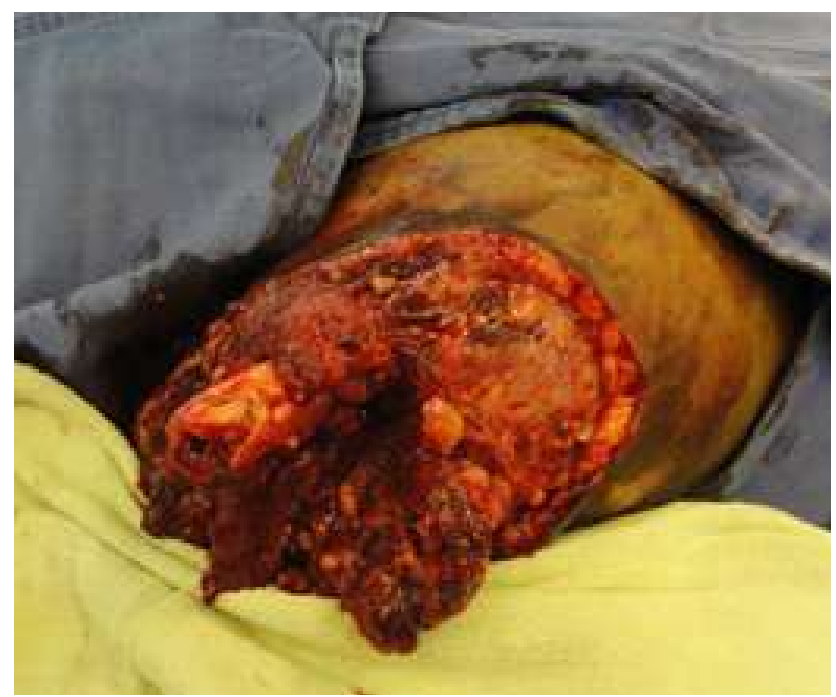

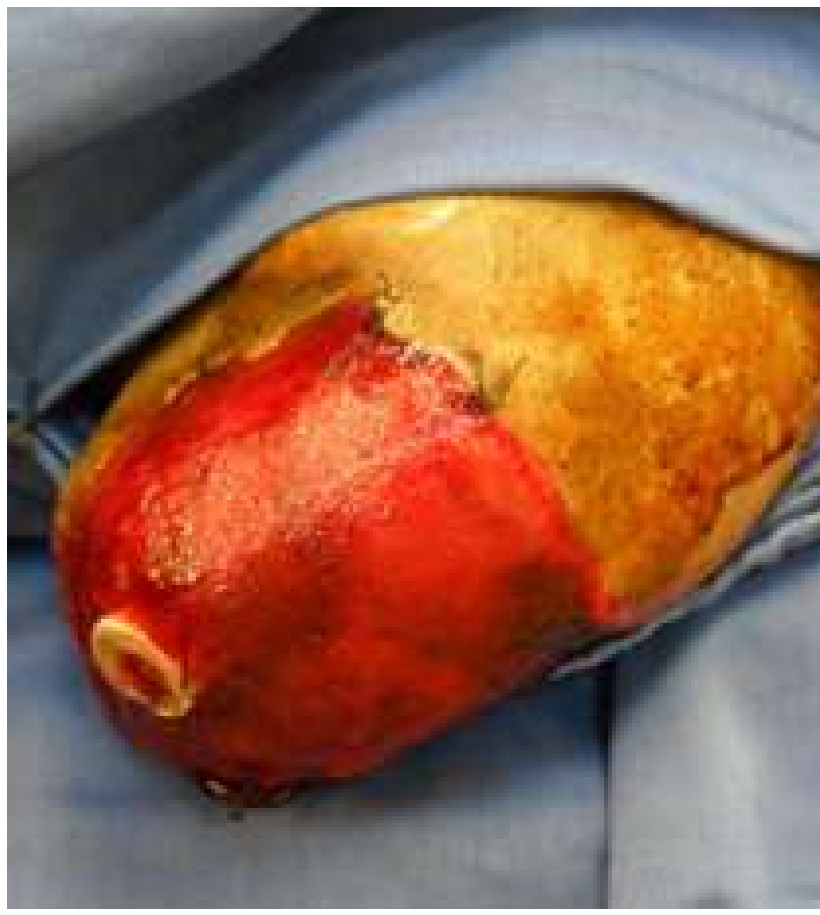

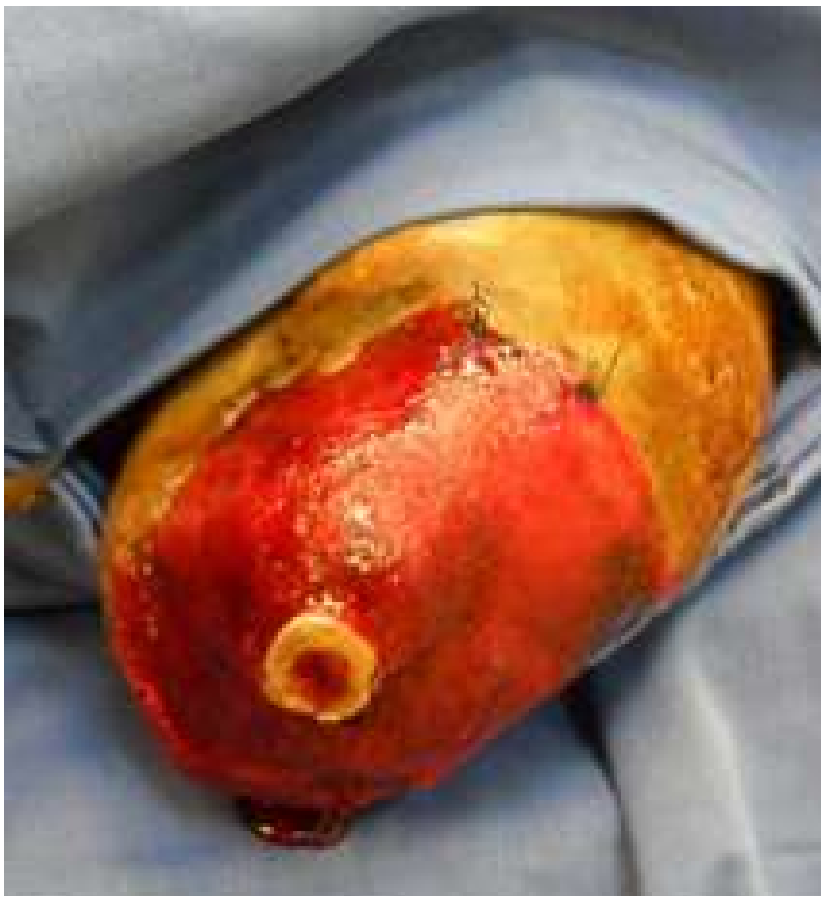

Foto 17

Foto 17: Imágenes de la lesión de brazo; a la izquierda la imagen post desbridamiento de la amputación. Las dos imágenes de la derecha son el resultado de 3 ciclos de sistema de cierre asistido al vacío con buen tejido de granulación. 


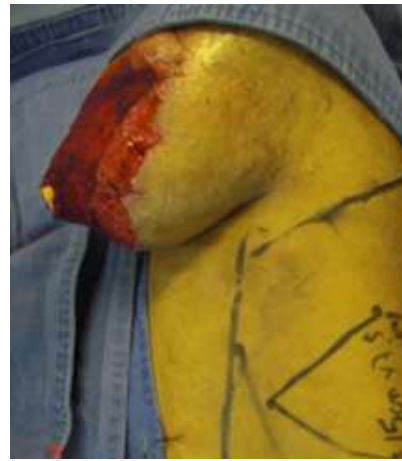

Foto 18

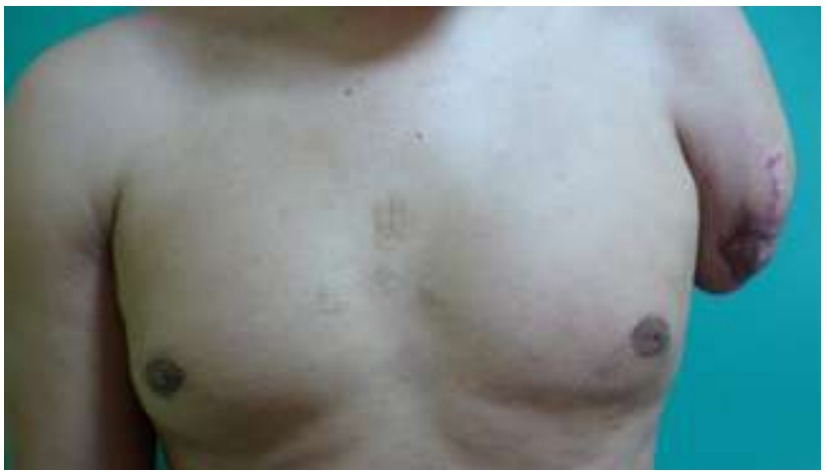

Foto 20

Foto 18,19 y 20 : Diseño del colgajo de dorsal ancho y resultado final de la extremidad amputada al cabo de 6 semanas post colgajo.

\section{CASO 4}

Paciente de 43 años de edad, con antecedente de quemadura por fuego en todas las extremidades, $16 \%$ SCT. En extremidad inferior izquierda la más comprometida, quemadura en toda la región posterior grado II profundo.

Se realizó escarectomía, estuvo 5 días con el sistema de cierre asistido al vacío, y luego de la terapia se realizó injerto de piel parcial, en la pierna, logrando la cobertura definitiva del defecto.

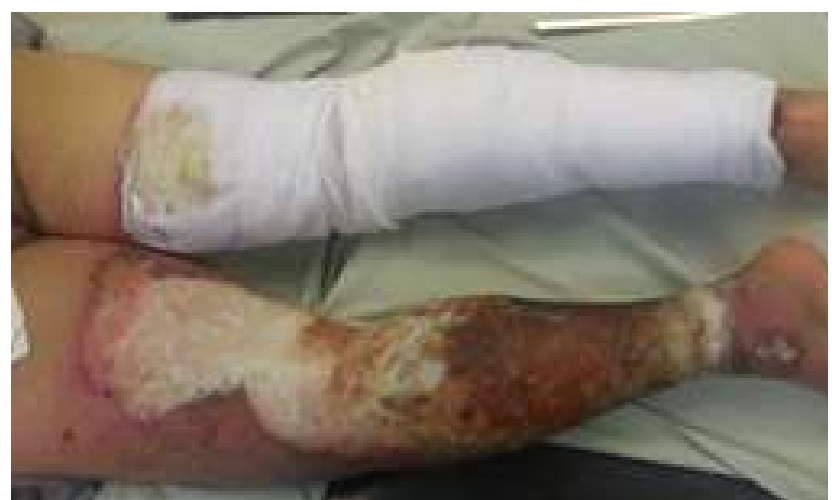

Foto 21

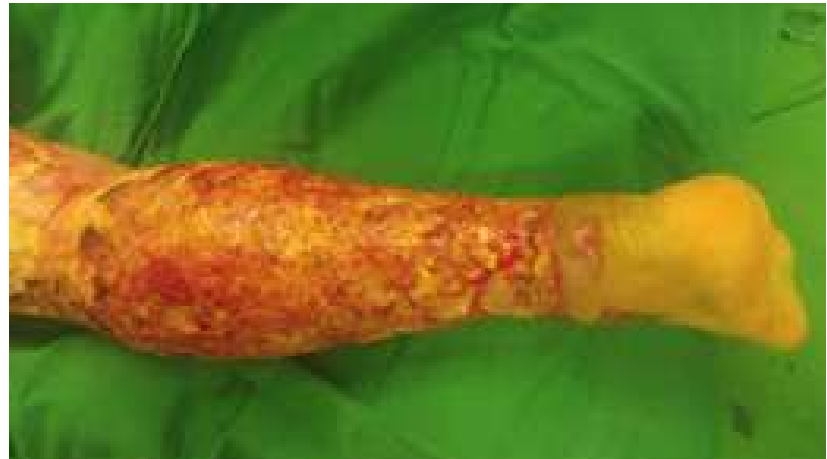

Foto 22

Foto ingreso 21 y 22 escarectomía.

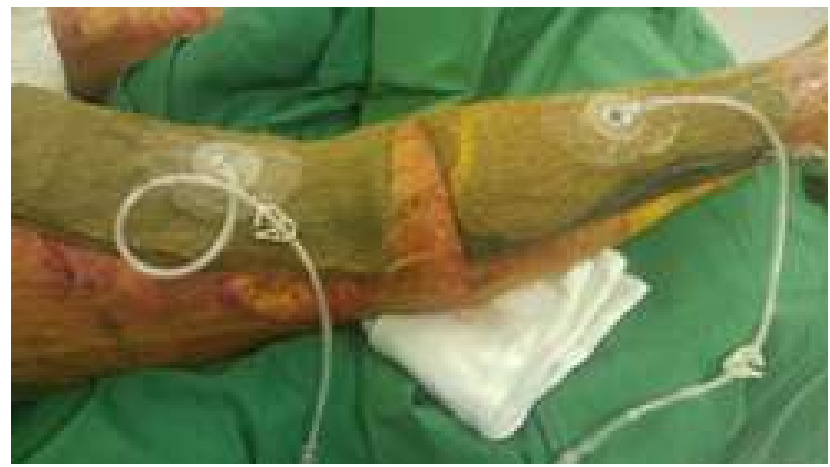

Foto 23

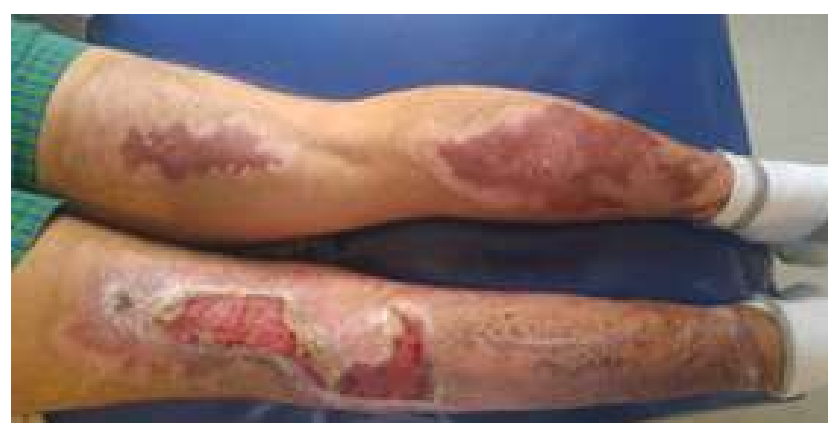

Foto 24

Foto 23 y 24 más cierre asisto al vacío e injerto.

\section{CASO 5}

Paciente de 52 años de edad, que fue intervenida de abdominoplastia 3 meses antes de que presentara la complicación (seroma y celulitis) con dehiscencia de la sutura.

Como antecedente de importancia tiene un S. de Raynaud.

Se administró tratamiento antibiótico IV y se realizaron dos limpiezas quirúrgicas en la segunda se decidió aplicar el sistema, una vez estuvimos seguros mediante una TC de que la pared ab- 
dominal se encontraba indemne y que el líquido que drenaba era de origen superficial, con el sistema estuvo 2 ciclos, al cabo de los cuales se realizó una segunda limpieza y cierre parcial de la herida, dejando un drenaje aspirativo por 5 días más.

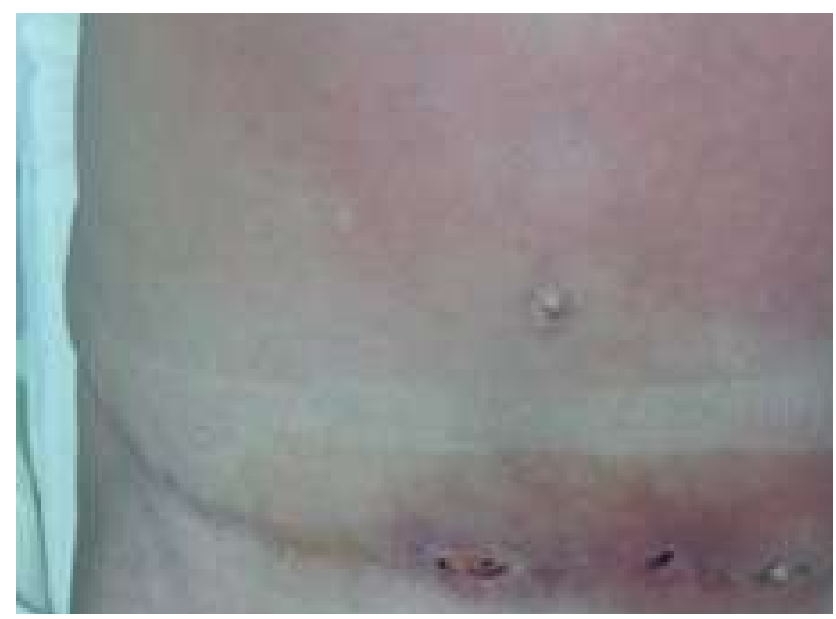

Foto 25

Foto 25 ingreso Celulitis

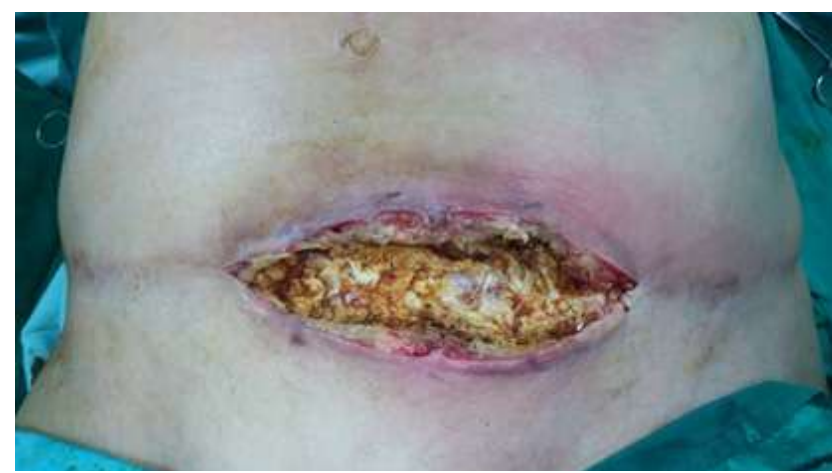

Foto 26

Foto 26 intraoperatorio, desbridamiento

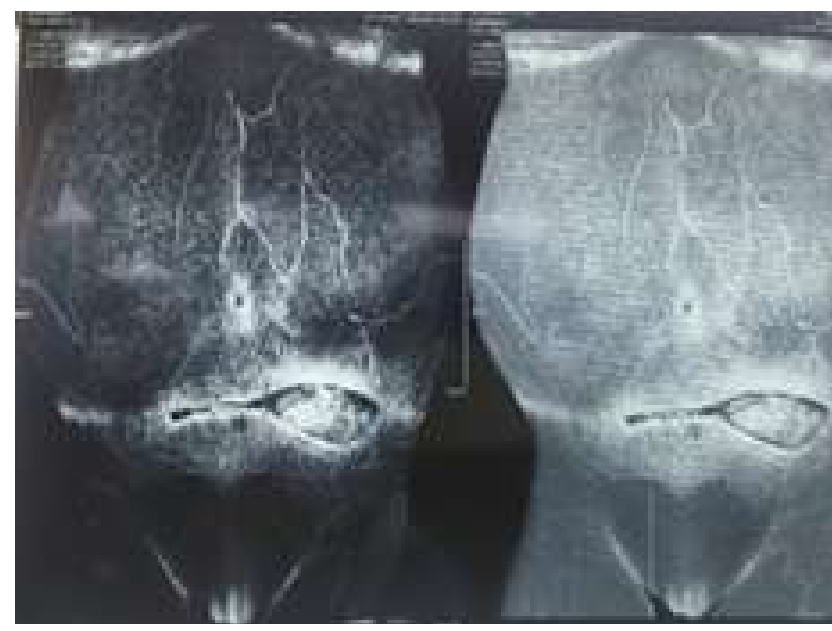

Foto 27

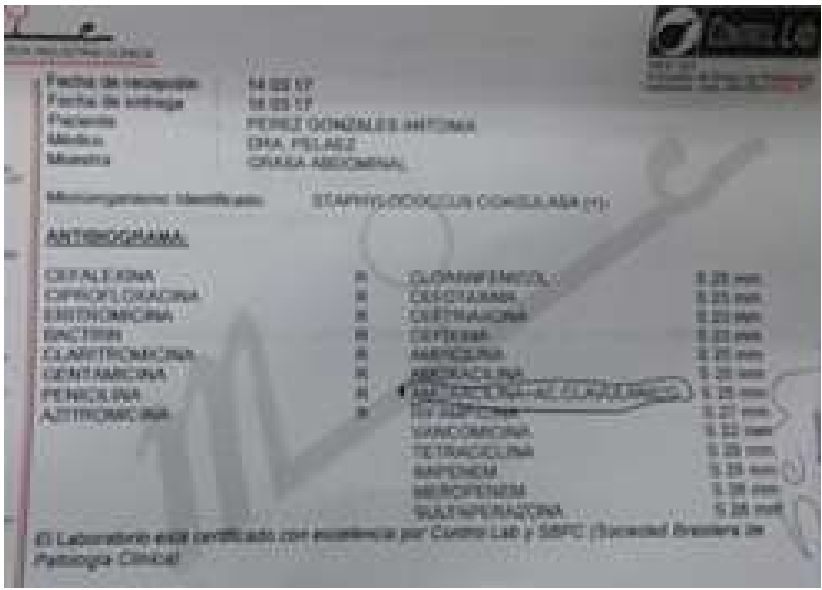

Foto 28

Foto 27 y 28 TC abdominal, y resultado de Cultivo y ATB

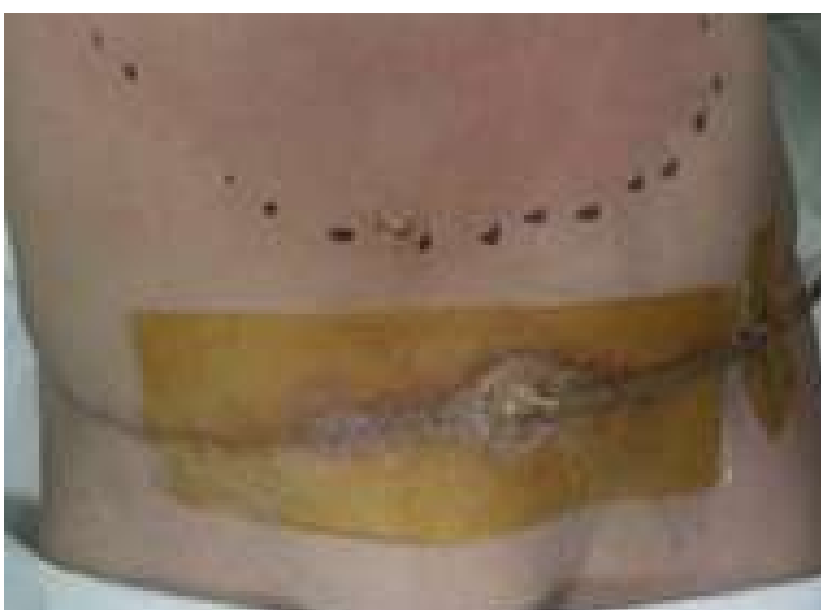

Foto 28

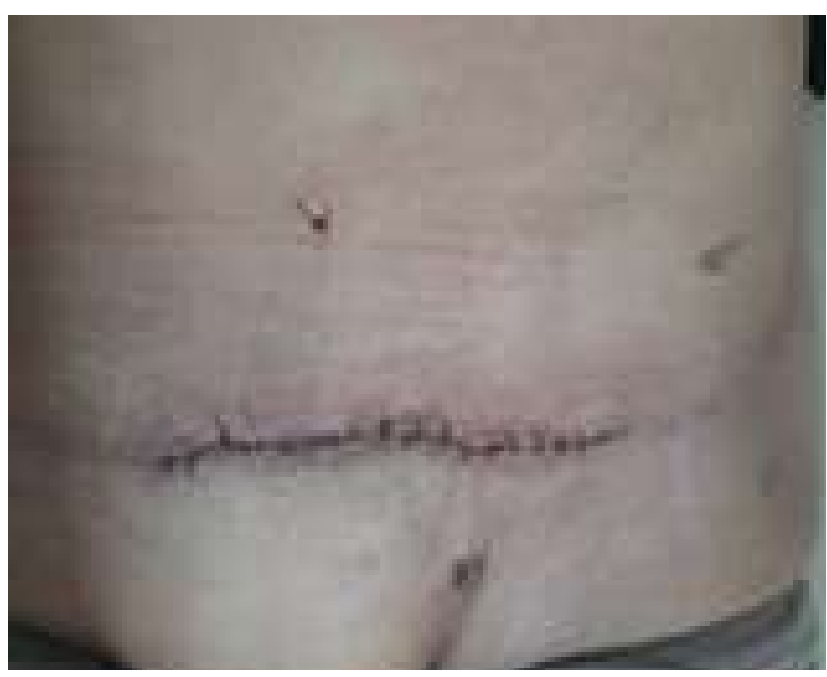

Foto 29

Foto 29 y 30 Aplicación del sistema y Postoperatorio de 2 semanas, cierre por primera de la herida, con sutura anti tensión. 


\section{DISCUSIÓN}

La finalidad del cierre asistido al vacío (C.A.V.) es acelerar el tiempo de cicatrización de la herida, protegerla de la contaminación externa y producir un efecto de limpieza mecánica; efectos logrados con el sellado de la herida y la succión que puede ser continua o intermitente.

\section{MECANISMO DE ACCIÓN}

Este sistema produce una succión sub-atmosférica que ayuda a remover el líquido y tejido necrótico, mejorando la perfusión vasculary disminuyendo la colonización bacteriana. 10,11

El efecto de la presión negativa mantiene una tensión sobre los tejidos de tal manera que logra aproximar los bordes de la misma, elimina el espacio muerto y ayuda a la formación de tejido de granulación.12

Es importante recalcar que con este sistema se disminuye notablemente la necesidad de curaciones frecuentes y desbridamientos bajo anestesia.

Un efecto secundario y positivo del sistema es la reducción de la inmunosupresión local, efecto logrado por el continuo drenaje de los líquidos de la herida en los que existe gran cantidad de mediadores solubles, incluyendo citoquinas proinflamatorias, las cuales han sido implicadas en la inmunosupresión que se presenta a menudo después de un trauma severo. Así, el sistema permite a los leucocitos infiltrar la herida y promover una reacción inmune eficiente contra la infección local. 13

La comparación con controles de heridas cubiertas por gasa impregnada con solución salina, demostró que mejoró significativamente la tasa de formación de tejido de granulación con la aplicación presión negativa continua $(63,3 \%$ y y con presión negativa intermitente (103\%).14,15

La observación de que el tratamiento intermitente parece más eficaz que el continuo es interesante. Existen dos posibles explicaciones (i) el ciclo intermitente da resultados de perfusión del tejido que se mantiene, porque el proceso de autorregulación capilar no está activado; y que (ii) las células que están en mitosis deben pasar por un ciclo de descanso, con los componentes celulares de producción y división, y la estimulación constante puede provocar que las células "ignoren" el estímulo $\mathrm{y}$, por tanto, se tornen ineficaces para la mitosis.

El ciclo intermitente permite la estimulación de las células con un tiempo para descansary así “prepararse” para el próximo ciclo. Por esta razón se sugiere que los ciclos de presión negativa deben utilizarse cíclicamente 16, 17.

Es indicado también que un período de 48 horas de aspiración continua al vacío, se puede aplicar para ejercer un rápido efecto de limpieza inicial.La presión negativa es una buena ayuda para el tratamiento de estas lesiones, pero no una panacea que reemplace otros principios básicos del tratamiento de las fracturas expuestas.

Dedmond et al 18 reportan 24 fracturas expuestas grado III que los cirujanos que atendieron el caso inicialmente (desbridación y fijación inicial), pensaron iban a terminar en procedimientos de cirugía reconstructiva mediante colgajos o transferencias de tejidos; de ellos sólo el 29\% de ésos pacientes requirieron éste procedimiento después de haber sido sometidos a este sistema, lo cual no solo es un éxito desde el punto de vista de complicaciones, sino una importante forma de reducir costes hospitalarios, y ello se acentúa más aún si se evita, como hacen muchos centros y también nosotros, que los cambios de la esponja se realice en la cama del paciente.

A través de este cuadro revisamos de forma práctica las indicaciones y contra indicaciones de uso para el sistema de cierre asistido al vacío

\section{CUADRO 1}

\section{INDICACIONES Y CONTRAINDICACIONES PARA EL USO DE VAC}

\begin{tabular}{|l|}
\hline Principales indicaciones son: \\
\hline Heridas traumáticas agudas \\
\hline Heridas subagudas (es decir, dehiscencias de heridas) \\
\hline Ulceras por presión \\
\hline Heridas crónicas (úlceras por estasis y úlceras diabéticas) \\
\hline Colgajos \\
\hline Coadyuvante en la integración de injertos de piel. \\
\hline Heridas posoperatorias complicadas infectadas \\
\hline Contraindicaciones : \\
\hline Tejido necrótico en escaras \\
\hline Osteomielitis (no tratada) \\
\hline Lesiones por cáncer. \\
\hline Alergia a los componentes del material \\
\hline Fístulas enterales \\
\hline Sangrado activo de un vaso o en napa \\
\hline
\end{tabular}

Existen, además, algunas precauciones como no colocar la esponja directamente sobre arterias o venas, ni en pacientes que estén usando anticoagulantes o con alteraciones en la coagulación. 


\section{CONCLUSIÓN}

Creemos que la terapia al vacío para cierre de heridas, ha demostrado mejorar de sobre manera la calidad de las heridas, en cuanto a que acorta tiempo en el que se encuentran aptas para la recepción de injertos, colgajos y/o cierre primario, también al mejorar el defecto a cubrir, permite que las cirugías reconstructivas necesarias para la cobertura de los mismos, sean menos complejas, lo que disminuye los riesgos y posibles complicaciones.

A pesar de no tener un grupo control, los resultados obtenidos son análogos a los reportados en la literatura mundial y con la experiencia adquirida durante este tiempo, para nosotros; los autores, la terapia VAC es nuestra primera elección, cuando nos enfrentamos a extremidades severamente lesionadas, heridas complejas y/ o crónicas.

\section{AGRADECIMIENTO}

Esta es una gran oportunidad; para agradecer al Dr. Martín Colombo, Cirujano Plástico Argentino, quien me transmitió sus conocimientos sin egoísmos, de tal manera que el aprender y seguir en la formación contínua en Cirugía Plástica se hace un placer. (Dra. A. Peláez).

Al Servicio de Ortopedia y Traumatología "Carlos E. Ottolenghi” del Hospital Italiano de Buenos Aires - Argentina, por las enseñanzas recibidas durante mi formación como especialista. (Dr. M. Paredes Núñez).

\section{BIBLIOGRAFÍA}

1. Fleischmann W, Strecker W, Bombelli M, Kinzl L. [Vacuum sealing as treatment of softtissue damage in open fractures]. Unfallchirurg 1993; 96(9): 488-92.

2. Fleischmann W, Lang E, Kinzl L. [Vacuum assisted wound closure after dermato fasciotomy of the lower extremity]. Unfallchirurg 1996; 99(4): 283-7.

3. Fleischmann W, Lang E, Russ M. [Treatment of infection by vacuum sealing].

Unfallchirurg 1997; 100(4): 301-4.

4. Muller G. [Vacuum dressing in septic wound treatment]. Langenbecks Arch ChirSupplKongressbd 1997; 114: 537-41.

5. Kovacs L, Kloppel M, Geishauser S, Schmiedl S, Biemer E. Vacuum sealing: a new andpromising regimen in the therapy of radiation ulcers. Br J Surgery 1998; 85: 70.

6. Brock WB, Barker DE, Burns RP. Temporary closure of open wounds: the vacuum pack. Am Surg 1995;61(1):30-34
7. Smith LA, Barker DE, Chase CW, Somberg LB, Brock WB, Burns RP. Vacuum pack technique of temporary abdominal closure: a four year experience. Am Surg 1997;63(12):1102-1107.

8. Barker DE, Kaufman HJ, Smith LA, Ciraulo DL, Richart CL, Burns RP. Vacuum pack technique of temporary abdominal closure: a 7 year experience with 112 patients. J Trauma Injury Infect Crit Care 2000;48(2):201-206

9. Argenta LC, Morykwas MJ. Vacuum-assisted closure: a new method for wound control and treatment: animal studies and basic foundation. Ann Plastic Surg 1997: 38(6):553-562.

10. Mullner T, Mrkonjic L, Kwasny O, Vecsei V. The use of negative pressure to promotethe healing of tissue defects: a clinical trial using the vacuum sealing technique. Br JPlastSurg 1997; 50(3): 194-9.

11. Banwell P, Withey S, Holten I. The use of negative pressure to promote healing.Br JPlastSurg 1998; 51(1): 79.

12. Morykwas MJ, David LR, Schneider AM, Whang C, Jennings DA, Canty C, Parker D, White WL, Argenta LC. Use of subatmospheric pressure to prevent progression of partial-thickness burns in a swine model. J Burn Care Rehabil 1999; 20(1): 15-21.

13. Gouttefangeas C, Eberle M, RuckP, etal. Functional Tlymphocytes infiltrate implanted polyvinyl alcohol foams during surgical wound closure therapy. Clin Exp Immunol 2001;124(3):398405

14. Zannis J. et al.: Compararison of Fasciotomy Wound Closures using traditional Dressing Changes and de Vacuum Assisted Closure Device. Ann Plast Surg 2009;62:407

15. Joseph E, Hamori CA, Bergman S, Roaf E, Swann NF, Anastasi GW. A prospective, randomized trial of vacuum-assisted closure versus standard therapy of chronic non-healing wounds. Wounds 2000; $12(2): 60-67$.

16. Collier. Know-how: A guide to vacuum-assisted closure (VAC). Nurs Times 1997;93(5): 32-3.

17. Morykwas MJ, Argenta LC. Nonsurgical modalities to enhance healing and care of soft tissue wounds South Orthop Assoc 1997; 6(4): 279-88.

18. Barnaby T. Dedmond, MD, Bill Kortesis, MD,Kathleen Punger, BA,Jordan Simpson, BS,Joseph Argenta, BA,Brenda Kulp, RN, BSN,Michael Morykwas, PhD, and Lawrence X. Webb, MD. The Use of Negative-Pressure Wound Therapy (NPWT) in the Temporary Treatment of Soft-Tissue Injuries Associated With High-Energy Open Tibial Shaft Fractures.J Orthop Trauma Volume 21, Number 1, January 2007 